\section{Role of human leukocyte antigens DRB1-DQB1 haplotype in the susceptibility to gastroesophageal reflux disease}

\author{
Batool Mutar Mahdi, ${ }^{1}$ \\ Riyadh Mohamad Hasan, ${ }^{2}$ \\ Wafaa Hazim ${ }^{1}$ \\ ${ }^{1}$ Department of Microbiology; \\ ${ }^{2}$ Department of Surgery, Al-Kindy \\ College of Medicine, University of \\ Baghdad, Iraq
}

\begin{abstract}
Gastro oesophageal reflux disease is due to involuntary gastric contents reflux into the esophagus from stomach, causing heartburn and acid regurgitation symptoms. Genetic and environmental factors are important factors in the causation of disease. Human Leukocyte antigens considered as an excellent marker for population genetics analysis and disease association. This study aimed to investigate the association between HLA-DRB1-DQB1 haplotype that inherited in linkage and its association with gastro oesophageal reflux disease (GERD). Patients and healthy controls were prospectively recruited from gastrocolonoscope unit at Al-Kindy Teaching Hospital (Baghdad-Iraq) between January and July 2016. Forty Iraqi Arab Muslims patients with a history of heartburn and dyspepsia compared with 100 Iraqi Arab Muslims control. All study patients and control group underwent upper gastroesophageal endoscope examination and HLA-DRB1 and HLA-DQB1 genotype were done using sequence spesific oligonucleotide primer to both groups. The frequencies of two haplotype HLA-DRB1/03-DQB1/03 and HLADRB1/13-DQB1/06 were significantly higher in patients with GERD while haplotype HLA-DRB1/03-DQB1/02 was significantly higher in control group. Sex had an effect in disease developing that haplotype HLADRB1/03-DQB1/03 was significantly more common in female patients that increased susceptibility to disease. This study identified that two haplotypes HLA-DRB1/03DQB1/03 and HLA-DRB1/13- DQB1/06 leads to increased susceptibility to GERD and haplotype HLA-DRB1/03-DQB1/02 was protective against GERD development.
\end{abstract}

\section{Introduction}

According to Genval workshop in 1999 that defined gastroesophageal reflux diseaseas (GERD) a disorder in the stomach which it's contents recurrently reflux into the lower part of the oesophagus causing heartburn and other symptoms. ${ }^{1}$ About one third of GERD patients had positive findings endoscopically and the rest had no obvious mucosal lesions., 2,3 The complications of GERD are Barrett's metaplasia that develops as a reaction to chronic damage by gastro-oesophageal reflux ${ }^{4}$ and oesophageal adenocarcinoma. $^{5}$ The development of these complications was due to altered immune outline in response to different stimulations of different antigens that modulate disease. ${ }^{6}$ The cytotoxic T lymphocyte and $\mathrm{T}$ helper cells can recognize these antigens presented by HLA (human leukocyte antigen) HLA-class I or HLA-class II molecules, respectively which plays an important role in immune response regulation. ${ }^{7}$ One study found that there is an association between HLA-DRB1 *15:01 and GERD in patients with $H$. pylori positive infection. ${ }^{8}$ Other study demonstrated that HLA B7 may increased susceptibility for Barrett's oesophagus. ${ }^{9}$ HLA-DR expression is found in the oesophageal tissue of patients with ongoing inflammation in the lamina propria and submucosa of the oesophageal as a result of cytokine release. ${ }^{10}$ The advantages of HLA class II expression on some tumor cells appears to restrict tumor growth and increased survival through stimulation of CD4 T helper cell response against tumor. ${ }^{11}$

This study aimed to investigate the association between HLA-DRB1-DQB1 haplotype that inherited in linkage and its association with gastro oesophageal reflux disease.

\section{Materials and Methods}

Patients with dyspepsia and heartburn and healthy controls were prospectively recruited from gastrocolonoscope unit at Al-Kindy Teaching Hospital in BaghdadIraq for the period between January and July 2016. The demographic details of all patients and control groups were recorded. Written informed consent was obtained from all patients and control group for this study. The study protocol was reviewed and approved by the Scientific and Ethical Committee of Al-Kindy medical college and Al-Kindy Teaching Hospital. The patient group and control groups were sex and age matched.

Forty Iraqi Arab Muslims patients with
Correspondence: Batool Mutar Mahdi, Department of Microbiology, Al-Kindy College of Medicine, University of Baghdad, Al-Nahda Square, Baghdad, Iraq.

Tel.: 00.964.1.077.02.553215.

E-mail: abas_susan@yahoo.com

Key words: Haplotypes; HLA; Gastro oesophageal reflux disease.

Contributions: BMM conducted the tests; RMH conducted the gastroscope exam; WH collected the data.

Conflict of interest: the authors declare no potential conflict of interest.

Funding: none.

Received for publication: 29 October 2017. Accepted for publication: 28 December2017.

This work is licensed under a Creative Commons Attribution NonCommercial 4.0 License (CC BY-NC 4.0).

(C) Copyright B.M. Mahdi et al., 2018 Licensee PAGEPress, Italy

Gastroenterology Insights 2018; 9:7471 doi:10.4081/gi.2018.7471

a history of heartburn and dyspepsia had been referred for upper gastrointestinal endoscopy at GIT center at Al-Kindy Teaching Hospital, Baghdad and a diagnosis of GERD were prospectively recruited. Those patients were compared with 100 Iraqi Arab Muslims control. All study patients and control group underwent upper gastroesophageal endoscope examination and HLA-DRB1 and HLA-DQB1 genotype were done using sequence spesific oligonucleotide primer to both groups.

Patients with Barrett's esophagus, esophageal varices, patients with secondary causes of gastro-oesophageal reflux disease, patients who had consumed antacids, H2 blockers, proton pump inhibitors, nonsteroidal anti-inflammatory drugs, alcohol, history of Helicobacter pylori eradication, subjects with a history of gastrointestinal surgery, peptic ulcer, and gastric cancer or with systemic disease requiring chronic medication were excluded from the study.

The control group was include 100 Iraqi Arab Muslims individuals undergoing upper gastrointestinal endoscopy for reasons other than reflux symptoms, Barrett's esophagus or any form of dyspepsia and heartburn. This group included those with normal OGD.

\section{Oesophagogastric examinations}

Both patients and control groups were 
Table 1. Frequencies of HLA-DRB1-DQB1 haplotypes in patients with GERD compared with healthy control subjects.

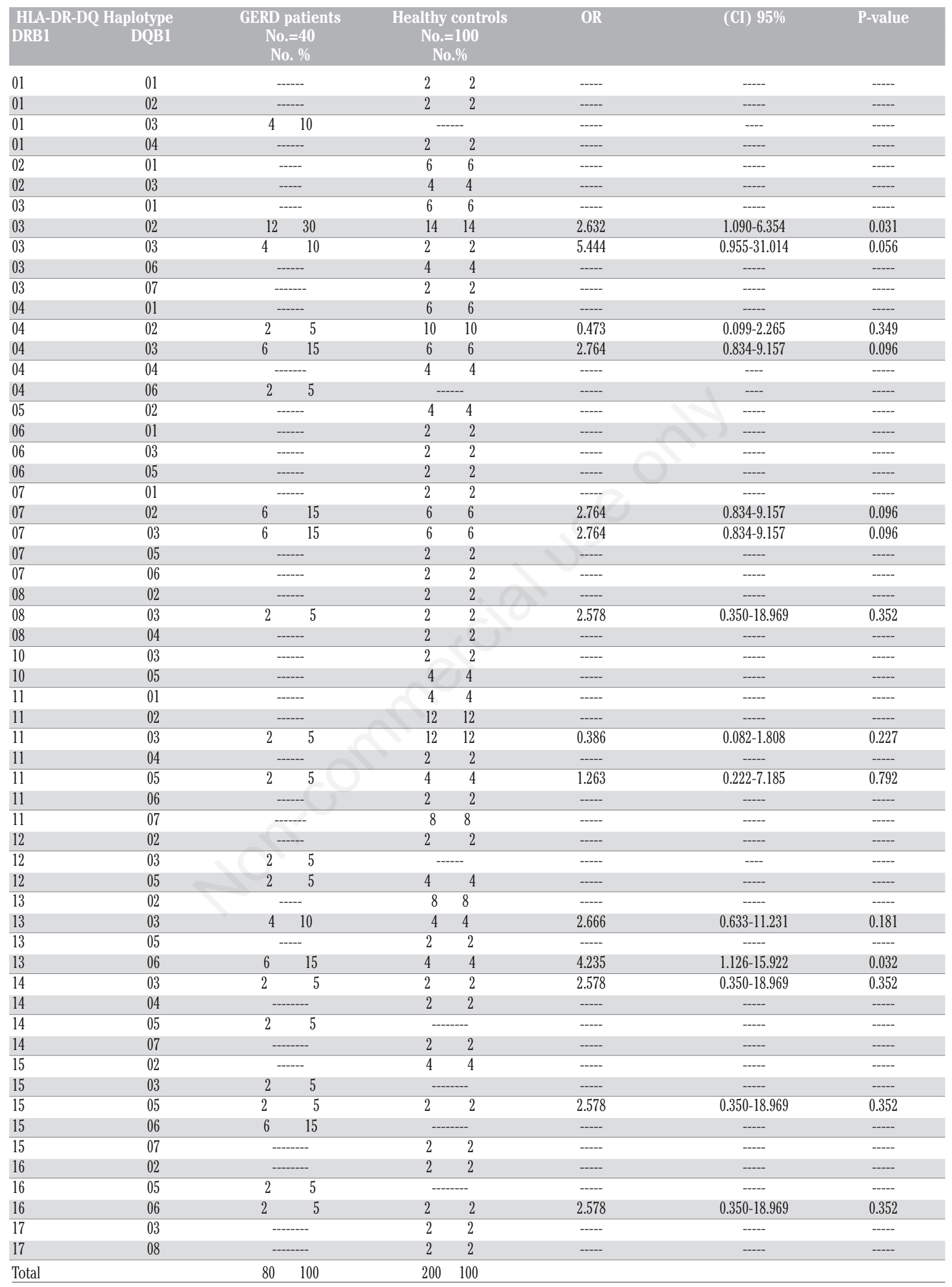


Table 2. Frequencies of HLA-DRB1-DQB1 haplotypes in male patients with GERD compared with healthy control subjects.

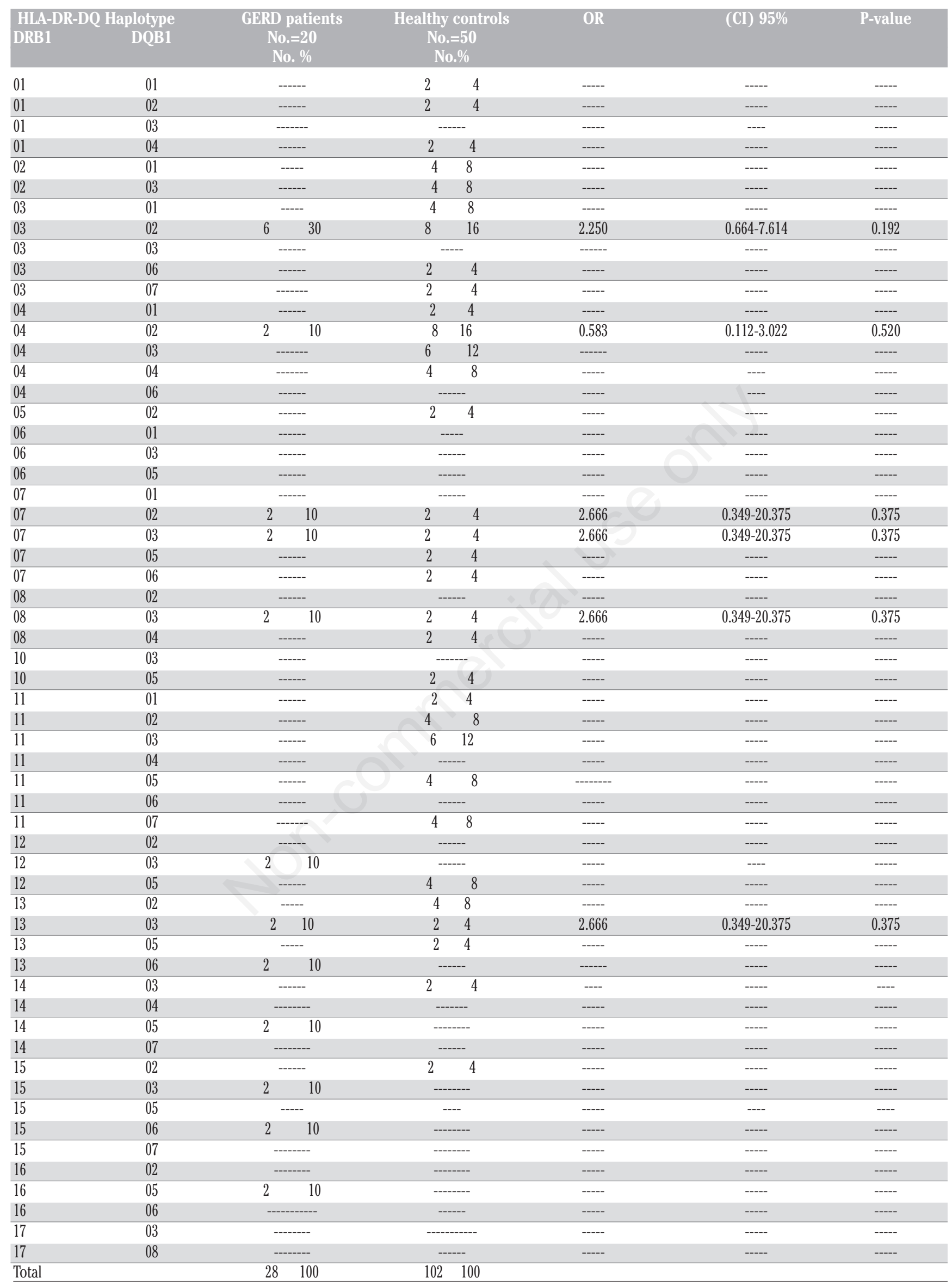


Table 3. Frequencies of HLA-DRB1-DQB1 haplotypes in female patients with GERD compared with healthy control subjects.

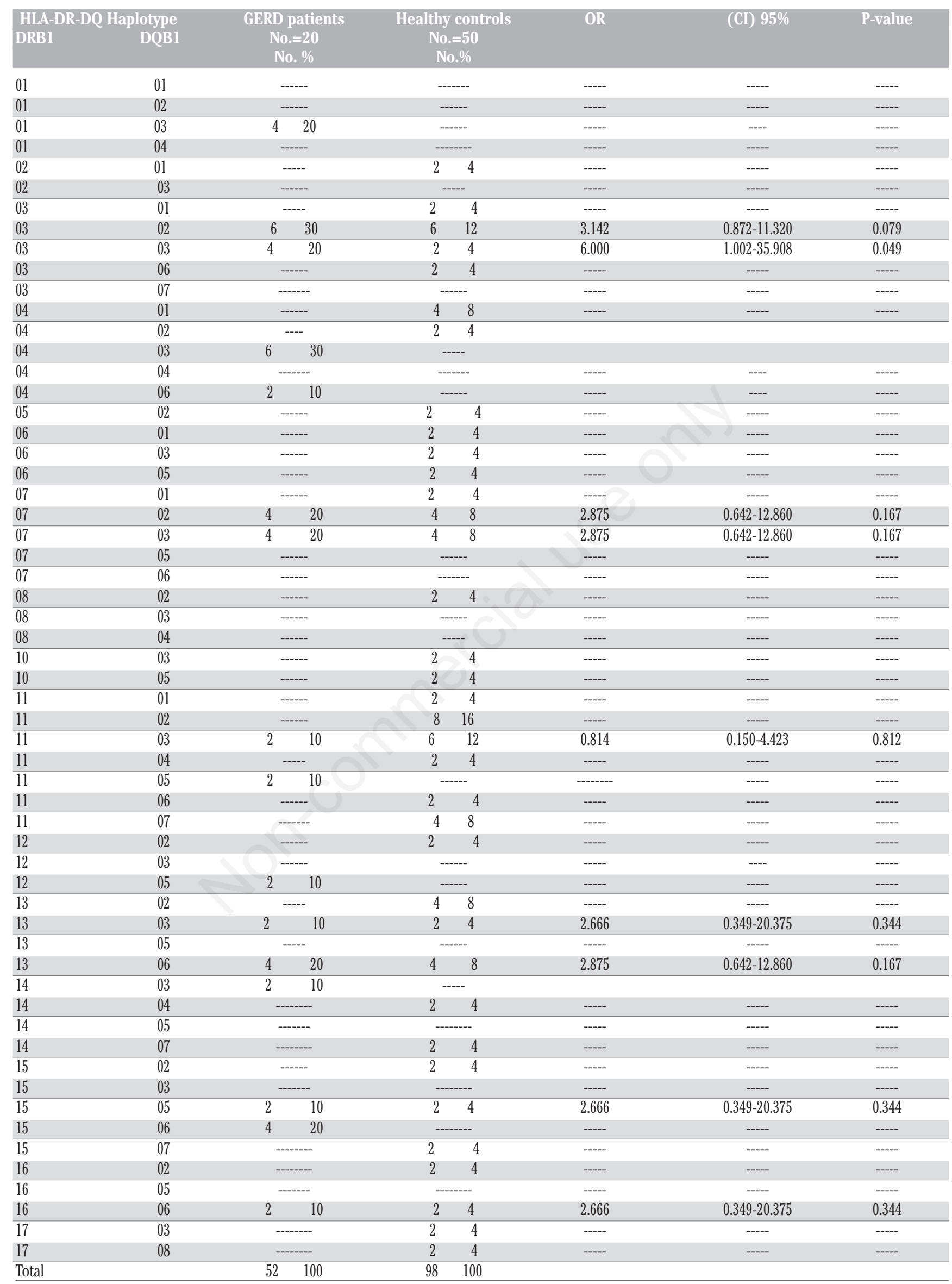


underwent upper gastrointestinal endoscopic examinations using gastroscope: GIFH260; Olympus, Tokyo, Japan and Display screen; Olympus OEV-261H liquid crystal display monitor; Olympus, Tokyo, Japan. The definition of gastroesophageal junction was the squamocolumnar junction and the proximal margin of gastric folds. According to Savary and Miller, $1979^{12}$ it was classified as Grade II which is confluent erosive or exudative mucosal lesions which do not extend around the entire esophageal circumference or Grade III which is erosive or exudative mucosal lesions which cover the entire esophageal circumference without stricture.

\section{HLA Class II genotyping (HLA- DRB1)}

DNA was extracted from human blood using blood kit (QIAmp DNA blood Mini Kit, QIAGEN INC- Germany). Then concentration and purification of DNA was estimated using Nanodrop -South Korea. DNA was verified by electrophoresis in a $2 \%$ agarose gel containing ethidium bromide and was visualized under UV light. Locus- and allele-specific amplification of genomic patients and control DNA was performed for DRB1 and DQB1. DNA Amplification and Hybridization was performed using a sequence-specific oligonucleotide (SSO) probes by HLA-DRB1 and HLA-DQB1 amplification and hybridization kits (SSO HLA type DRB1 plus and Mastermix for HLA type DRB1 Amp plus kits -Innogenetics-Belgium) and (SSO HLA type DQB1 plus and Mastermix for HLA type DQB1 Amp plus kits -InnogeneticsBelgium) by AutoLipa - 48InnogeneticsBelgum. The results were interpreted using LiRas version-5.0 software- InnogeneticsBelgium.

\section{Statistical analysis}

HLA-DRB1 and HLA-DQB1 frequencies were determined by direct counting. The frequency of each allele was compared between patients and control group using chi-square test Fisher exact test using MiniTab version. 3.0 software. In each comparison, the Odds ratio (OR) along with the $95 \%$ confidence interval $(95 \% \mathrm{CI})$ was used. Gene frequencies for both groups were calculated. $P$-value less than 0.05 were considered statistically significant.

\section{Results}

The frequencies of two haplotype HLADRB $1 * 03-\mathrm{DQB} 1 * 03$ and HLA-DRB*13DQB $1 * 06$ were significantly higher in patients with GERD while haplotype HLADRB $1 * 03-D Q B 1 * 02$ was significantly higher in control group Table 1. Sex had an effect in disease developing that haplotype HLA-DRB $1 * 03-D Q B 1 * 03$ was significantly more common in female patients that increased susceptibility to disease as shown in Tables 2 and 3.

\section{Discussion}

GERD is an important disease that leads to Barrett's oesophagus and malignant transformation of the esophagus. ${ }^{13}$ This study shown that the expression of two haplotype HLA-DRB1*03-DQB1*03 and HLA-DRB1*13-DQB1*06 were significantly higher in patients with GERD while haplotype HLA-DRB $1 * 03-\mathrm{DQB} 1 * 02$ was significantly higher and protective in control group. The HLA had a central role in T cell or NK cell recognition and in patients who had other alleles leads to evasion of immune observation by metaplastic cells, that's leads to Barrett's metaplasia-dysplasia-adenocarcinoma sequence; so HLA class I (HLA-ABC) expression is down regulated and class II (HLADP, DQ, DR) expression is up regulated in Asian patients with Barrett's oesophagus that leads to malignant transformation of the epithelial cells using immunohistochemical staining. ${ }^{14}$ Additional studies are necessary to assess the value of these genetic aberrations and related molecular changes in disease prognosis. HLA expression may help to stratify patients into low risk and high risk sets for GERD development. The Indian Asian patients showed that HLA-B7 had an association with GERD disease. ${ }^{15}$ The expression of HLA-DR have increased on eosinophils in patients with eosinophilic esophagitis. ${ }^{16}$ Thus, polymorphism in HLA system is an excellent marker for population genetic analyses and disease-association studies. There is a racial difference in GERD disease prevalence due to presence of many genes that affect the disease development. ${ }^{17,18}$ Mahdi et al., ${ }^{19}$ reported that HLA-DRB1 *15:01 had an association with GERD patients infected with $H$. pylori. Regarding recombination that is most commonly occurs in or around genes and haplotyoe structure strongly affects synapsis will occur that facilitates subsequent recombination events to occur. ${ }^{20,21}$ The differences in the linkage may be due to synaptonemal complex, which is a proteinaceous structure formed by transverse filaments and lateral elements. At some stage in prophase of meiosis I, the link between 30 near gene region and 50 near gene region may be small in space. The block of this investiga- tion is merely a part of 1 larger human block, but self-similar phenomenon exists extensively in biology. This study is the first to report on the genetics of HLA haplotype polymorphisms in Iraqi Arab Muslims population. We got results from the low-frequency variants of the HLA haplotypes that are more powerful to detect risk and to detect protective HLA haplotype variants in GERD patients.

\section{Conclusions}

This study identified that two haplotypes HLA-DRB1/03-DQB1/03 and HLADRB1/13- DQB1/06 leads to increased susceptibility to GERD and haplotype HLADRB1/03-DQB1/02 was protective against GERD development.

\section{References}

1. Dent J, Brun J, Fendrick AM, et al. On behalf of the Genval Workshop Group. An evidence-based appraisal of reflux disease management - the Genval Workshop Report. Gut 1999;44:S1-16.

2. Martinez SD, Malagon IB, Garewal HS, et al. Non-erosive reflux disease (NERD) - acid reflux and symptom patterns. Aliment Pharmacol Ther 2003;17: 537-45.

3. Fujiwara Y, Higuchi K, Shiba M, et al. Differences in clinical characteristics between patients with endoscopy-negative reflux disease and erosive esophagitis in Japan. Am J Gastroenterol 2005;100:754-8.

4. Jankowski J. Gene expression in Barrett's mucosa: acute and chronic adaptive responses in the oesophagus. Gut 1993;34:1649-50.

5. Hameeteman W, Tytgat GNJ, Houthoff HJ, et al. Barrett's esophagus: development of dysplasia and adenocarcinoma. Gastroenterology 1989;96:1249-56.

6. Fitzgerald RC, Onwuegbusi BA, BajajElliott M, et al. Diversity in the oesophageal phenotypic response to gastro-oesophageal reflux: immunological determinants. Gut 2002;50:451-9.

7. Townsend A, Ohlen C, Bastin J, et al. Association of class I major histocompatibility heavy and light chains induced by viral peptides. Nature 1989;340:443-8.

8. Mahdi BM, Hasan RM, Salih WH. Human leukocyte antigen HLADRB1 determinants susceptibility to gastroesophageal reflux disease .Arq Gastroenterol 2017;54:41-5.

9. Lopez Nevot MA, Esteban F, Ferron A, 
et al. HLA class I gene expression on human primary tumours and autologous metastases: demonstration of selective losses of HLA antigens on colorectal, gastric and laryngeal carcinomas. $\mathrm{Br} \mathrm{J}$ Cancer 1989;59:221-6.

10. Oberrhuber G, Puspok A, PeckRadosavlevic $M$, et al. Aberrant esophageal HLA-DR expression in a high percentage of patients with Crohn's disease. Am J Surg Pathol 1998;22:383-90.

11. Lazaris AC, Theodoropoulos GE, Davaris PS, et al. Heat shock protein 70 and HLA-DR molecules tissue expression. Prognostic implications in colorectal cancer. Dis Colon Rectum 1995;38:739-45.

12. Savary M, Miller G. The Esophagus. Handbook and Atlas of Endoscopy. Solothurn: Gassmann Verlag AG; 1978.

13. Hameeteman W, Tytgat GNJ, Houthoff HJ, van den Tweel JG. Barrett's esoph- agus: development of dysplasia and adenocarcinoma. Gastroenterology 1989;96:1249-56.

14. Rajendra S, Ackroyd R, Karim N, et al. Loss of human leucocyte antigen class I and gain of class II expression are early events in carcinogenesis: clues from a study of Barrett's oesophagus. J Clin Pathol 2006;59:952-7.

15. Rajendra S, Ackroyd R, Murad S, et al. Human leucocyte antigen determinants of susceptibility to Barrett's oesophagus in Asians - a preliminary study. Aliment Pharmacol Ther 2005;21:1377-83.

16. Patel AJ, Fuentebella J, Gernez Y, Nguyen T, Bass D, Berquist W, Cox K, Sibley E, Kerner J, Nadeau K. Increased HLA-DR expression on tissue eosinophils in eosinophilic esophagitis. J Pediatr Gastroenterol Nutr 2010;51:290-4.

17. Rajendra S, Alahuddin S. Racial differences in the prevalence of heartburn.
Aliment Pharmacol Ther 2004;19:3756.

18. Mohammed I, Cherkas LF, Riley SA, et al. Genetic influences in gastrooesophageal reflux disease: a twin study. Gut 2003;52:1085-9.

19. Mahdi BM, Hasan RM, Salih WH. Human leukocyte antigen HLADRB1 determinants susceptibility to gastroesophageal reflux disease. Arq Gastroenterol 2017;54:41-5.

20. He L, Dooner HK. Haplotype structure strongly affects recombination in a maize genetic interval polymorphic for Helitron and retrotransposon insertions. Proc Natl Acad Sci USA 2009;106: 8410-6.

21. Fraune J, Schramm S, Alsheimer M, et al. The mammalian synaptonemal complex: protein components, assembly and role in meiotic recombination. Exp Cell Res 2012;318:1340-6. 